\title{
Application of Virtual Instrument in the Plunger Pump Vibration Test
}

\author{
Zhengyi ZHANG ${ }^{1, a)}$, Chuntong LIU' ${ }^{1)}$, Hongcai $\mathrm{LI}^{1)}$, Zhenxin $\mathrm{HE}^{1)}$, Xiaofeng \\ $\mathrm{ZHAO}^{1)}$, and Jianfeng $\mathrm{YU}^{2)}$ \\ ${ }^{1}$ The Rocket Force University of Engineering, Xi'an 710025,China \\ ${ }^{2}$ The Integrated Planning Division of the Military Representative Office of The Rocket Force in \\ Chongqing, Chongqing 404100,China \\ a)18809231139@163.com
}

\begin{abstract}
The vibration of plunger pump to produce the serious influence its service life, through the plunger pump vibration test of the testing system can understand the vibration of the plunger pump, analysis to know the source of the harmful vibration, thus to find a way to eliminate its impact, has important significance to improve the quality of printing quality. In order to study of plunger pump shell vibration, get a reliable vibration test data, at the same time for traditional plunger pump vibration test methods required for equipment is more and more complex, high cost and poor reliability faults, based on virtual instrument technology, application of LabVIEW graphical, modular programming language to write the data handler, formed a plunger pump shell vibration test system. In detail elaborated the system hardware structure and software structure of the composition, function and design idea and theoretical basis, and use the system to test the plunger pump vibration signal acquisition and processing data of FFT, the measured data of frequency domain information, it is concluded that the vibration signals of the plunger pump.
\end{abstract}

\section{INTRODUCTION}

Plunger in the running process produces vibration phenomenon. Vibration will not only affect the service life of pump and efficiency, and reduces plunger pump operation safety and reliability. Plunger pump vibration can be caused by many reasons, in order to study the law of the pump shell vibration, find that causes the vibration of the factors and the relationship between these factors and the vibration rule, to accurately obtain the plunger pump shell vibration frequency, velocity and displacement characteristics, especially the characteristics of the spectrum. Among them, the vibration frequency is a result of the combined action of various forces, can reflect the variation of the pump body stress instantaneous. This amount does not need the accumulation of time, so responsive, can reflect the real-time state of vibration. This paper established the plunger pump flow induced vibration test and analysis system for the characteristics of vibration frequency as the measurement and analysis. Many scholars at home and abroad are also built a vibration test system of plunger pump vibration signal measurement and research. NiYongYan using measured vibration signal analysis to diagnose the process such as the operation condition of the pump [1]; Behzad $\mathrm{M}$ etc. Through the collection and analysis of vibration signal fault diagnosis of plunger pump [2]. System in this paper, the vibration frequency of the test is mainly directed against the plunger pump shell vibration is designed, the first design the FBG vibration sensor target 
type, through the optical fiber demodulation instrument for fiber optic center wavelength) (that is, the vibration signal acquisition, provide the basis for subsequent analysis of shell vibration rule. In the traditional vibration test system, the instrument complex, poor reliability test results, the cost is higher, is restricted by a lot of in the practical application. Appeared with the development of computer technology, virtual instrument technology is different from the traditional instruments, it can through different testing function software module and a variety of measuring instruments of the combination between the hardware to realize multiple test function, and high accuracy, good stability, can build a good performance and low cost of the test system, in terms of mechanical test and test application is very extensive [3]. Based on virtual instrument, this paper builds on plunger pump flow induced vibration test system, and the test piston pump were measured.

\section{VIBRATION TEST SYSTEM HARDWARE STRUCTURE}

The system hardware is mainly composed of two parts: FBG target type vibration sensor and data acquisition processing system.

\subsection{FBG target type vibration sensor}

By a fiber Bragg grating strain sensing properties ,we can know[4],

$$
\frac{\Delta \lambda_{B}}{\lambda_{B}}=\left(1-P_{e}\right) \varepsilon_{X}
$$

${ }_{\Delta} \lambda_{B}$ as the center wavelength variation, $\lambda_{B}$ as the center wavelength, Pe valid elastic-optic coefficient, axial strain to the outside world.

The FBG strain sensor model shows that if the FBG strain he felt for the dynamic strain of periodic change over time, and the change is due to the vibration of the objects in connection with the, you can use this model to measure the cyclical vibration (such as harmonic vibration).

Designed as shown in figure 1 FBG target type vibration sensor, the size is shown in figure 2.

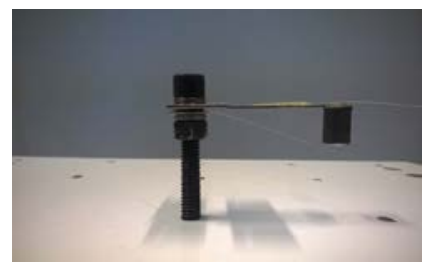

Figure 1. FBG target type vibration sensor

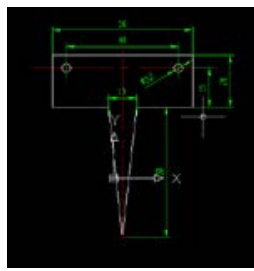

Figure 2. Model size

\section{2 data collection and processing system}

At present, the FBG sensing signal demodulation scheme for a variety of, according to the working principle of the modem one in general can be divided into: spectrometer analysis and method of edge filter, tunable filter method, interference scanning method, such as different demodulation methods have their respective features and applicable scope[5]. Using piezoelectric ceramics (PZT) consisting of tunable Fabry - Perot cavity (F-P) measurement center wavelength of the fiber Bragg grating sensor, can be directly convert wavelength signals into electrical signals, has a large scanning range, small volume, low price advantages, and the range of scanning can be according to the actual application situation carries on the corresponding design[6], therefore, tunable Fabry - Perot filter method is a better demodulation scheme, the system adopts the scheme. 


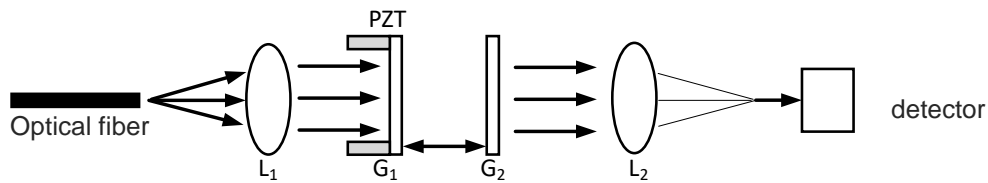

Figure 3. Tunable Fabry - Perot cavity structure theory

F-P cavity on the function of a narrow-band filter [7], a band of parallel light incident to F-P cavity, only can satisfy the coherent condition of certain wavelengths of light interference occurs, produce coherent greatly, tunable F-P filter method is using the characteristics of F-P cavity of the fiber Bragg grating wavelength demodulation

\section{VIBRATION TEST SYSTEM SOFTWARE DESIGN}

Using LabVIEW software modularization program design idea and the way of graphical programming, this paper developed a plunger pump vibration test and analysis system. Vibration test and analysis system with the traditional test method of difference is that using the virtual instrument instead of the traditional log analysis equipment. (the so-called Virtual Instrument of Virtual Instrument, VI for short), is on the computer as the core of hardware platform, design defined by the user, which has the function of Virtual panel and test analysis computer Instrument system, its main functions include data acquisition, signal processing and data storage and output. Figures 4 and 5, respectively, for the plunger pump vibration test system user login interface and the user login procedure diagram, figure 6 as plunger pump vibration test system application.

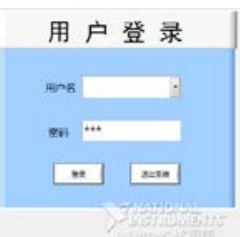

Figure 4. Plunger pump vibration test system user login interface

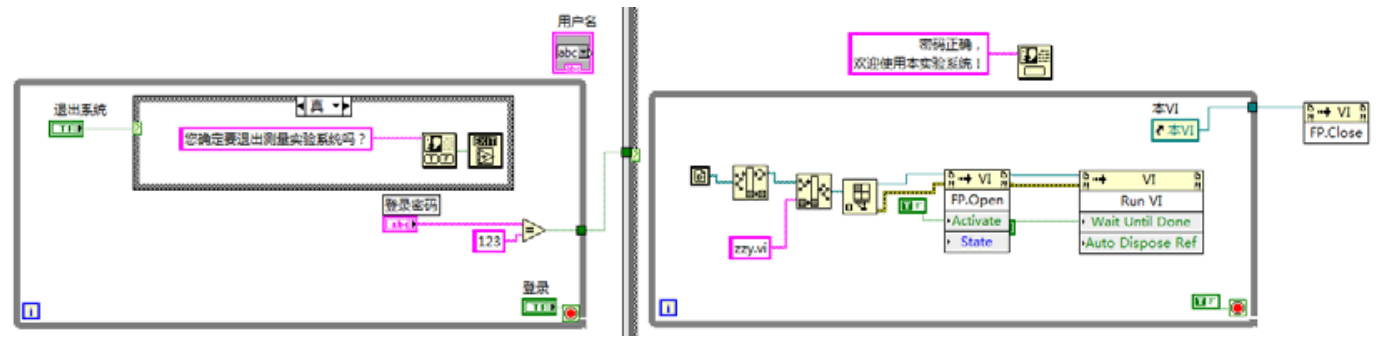

Figure 5. Plunger pump vibration test system user login procedure chart

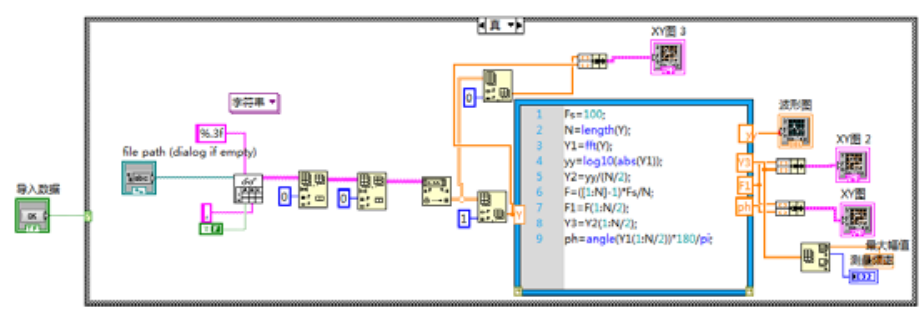

Figure 6. Plunger pump vibration test system program 


\section{VIBRATION TEST SYSTEM TEST}

Placed with vibration test system in vibration platform, set up the vibration frequency of $35 \mathrm{~Hz}$, run the vibration test system, as shown in figure 7, due to the resonance frequencies of the vibration table is put desktop, there exists a $5 \mathrm{~Hz}$ low frequency interference signal. Measure the frequency of $36.5 \mathrm{~Hz}$, error within the acceptable range, meet the requirements of industrial measurement.

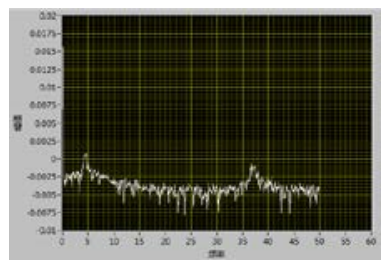

Figure 7. Frequency curve

\section{CONCLUSION}

This system give full play to the advantages of virtual instrument, first of all, it USES less hardware, greatly reduce the development time and cost, due to the limitations of the hardware, can be easily on every position of the engine vibration measured; Second, the system USES the computer a powerful user interface, data collection and analysis, with which to implement the plunger pump vibration test.

\section{ACKNOWLEDGMENTS}

This work was supported by the National Natural Science Foundation of China (No.41404022) and the Shanxi National Science Foundation (No.2015JM4128)

\section{REFERENCES}

1. Behzad M, Bastami A R, Maassoumlan M. Fault diagnosis of a centrifugal pump by vibration analysis[C]MProceedings of the 7th Biennial Conference on Engineering Systems Design and Anal-ysis.2004:221 -226.

2. Jong-Soo Choi, Dennis K McLaughlin, Donald E Thompson. Experiment on the unsteady flow field and noise generation in a centrifugal pump impeller[J].Journal of Sound and Vibration,2003 (263):493 -514.

3. National Instruments LabVIEW He1p[Z]. Texas: National Instruments. 2003.

4. MCCLELLAN JH, PARKS TW. A Unified Approach to the Design of Optimum FIR Linear Phase [J]. IEEE Trans on Circuit Theary,1973, 20(6): 697 -701.

5. LabVIEW Function and VI Reference Manual[Z]. National Instruments Corporation 1998.

6. Advantech Corporation. Device Driver.s Manual for USB-4711A[Z].

7. National Instruments DataSocket Technical Overview[Z]. Texas: National Instruments. 1998. 